\title{
GCU
}

Glasgow Caledonian

University

University for the Common Good

\section{Electrocardiogram-based feature extraction for machine learning classification of obstructive sleep apnea}

Mitiche, Imene; Morison, Gordon; Stewart, Brian G.

Published in:

International Journal of Signal Processing Systems

DOI:

10.18178/ijsps.4.6.515-518

Publication date:

2016

Document Version

Publisher's PDF, also known as Version of record

Link to publication in ResearchOnline

Citation for published version (Harvard):

Mitiche, I, Morison, G \& Stewart, BG 2016, 'Electrocardiogram-based feature extraction for machine learning classification of obstructive sleep apnea', International Journal of Signal Processing Systems, vol. 4, no. 6, pp. 515-518. https://doi.org/10.18178/ijsps.4.6.515-518

\section{General rights}

Copyright and moral rights for the publications made accessible in the public portal are retained by the authors and/or other copyright owners and it is a condition of accessing publications that users recognise and abide by the legal requirements associated with these rights.

Take down policy

If you believe that this document breaches copyright please view our takedown policy at https://edshare.gcu.ac.uk/id/eprint/5179 for details of how to contact us. 


\title{
Electrocardiogram-Based Feature Extraction for Machine Learning Classification of Obstructive Sleep Apnea
}

\author{
Imene Mitiche, Gordon Morison, and Brian G. Stewart \\ Glasgow Caledonian University, Glasgow, Scotland \\ Email: imitic200@caledonian.ac.uk, \{Gordon.morison, b.stewart\}@gcu.ac.uk
}

\begin{abstract}
This paper introduces a new feature extraction technique based on Time Sequence Analysis, combined with machine learning classification technique called Extreme Learning Machine (ELM), for automatic diagnosis of Obstructive Sleep Apnea (OSA) syndrome. The feature was extracted from Electrocardiogram (ECG) signal of patients with and without OSA. The ECG recordings were labelled as "Apnea" or "Normal" by experts' examination. The data was freely available online from Physionet database. The feature extraction and classification algorithms were implemented on Matlab environment and the performance was evaluated in terms of OSA detection accuracy percentage. The aim of the study is to provide a low computational feature extraction technique for automatic OSA diagnosis. Simulation results show that OSA detection with $80.3 \%$ accuracy is possible using one feature only. It is concluded that the proposed technique offers OSA diagnosis with good enough OSA detection while reducing computation.
\end{abstract}

Index Terms-Obstructive Sleep Apnea (OSA), Time Sequence Analysis (TSA), Extreme Learning Machine (ELM), detection accuracy

\section{INTRODUCTION}

Obstructive Sleep Apnea (OSA) is a common perturbation in sleep due to the inability of breathing which is caused when the upper airway closes completely [1]. It was reported that OSA increases the risk of type II diabetes [2], susceptibility to accidents and neurocognitive dysfunction. Furthermore, this syndrome increases the risk of acute stroke and yields to many more diseases [3]. Besides, OSA affects the daily lifestyle of the individuals, through tiredness causing low performance in their daily activities, since the individual suffers from interrupted sleep in order to breathe. Patients could be treated earlier and these consequences would be avoidedif OSA is detected or predicted at early stage. Traditional methods for OSA detection make use of Polysomnography (PSG) to obtain a set of signals including Electroencephalograph (EEG), respiratory movement, oral and nasal respiration, and oxygen saturation signal. Such technique is expensive, it requires more: sleep centers, pecular data acquisition systems and

Manuscript received November 16, 2015; revised June 17, 2016. specialised personnel with OSA diagnosis expert knowledge. These inconvenients are encouragement to develop simpler and cheaper OSA diagnosis techniques. A number of automatic and low cost OSA detection methods have been proposed in Computers in Cardiology (CinC) challenge based on ECG waveform analysis and a comparison of their performance is discussed in [4]. The latter represents heart activity andconsists of five components P, Q, R, S and T. The relation between ECG waveform and OSA can be observed in hear rate variations i.e. the heart rate decreases during OSA events due to lack of inhaled oxygen [5]. The aim of this work is to develop a novel and reliable feature extraction algorithm with low computation complexity for automatic OSA diagnosis based on ECG recordings. The process involves three main steps: computing one feature from each minuteof ECG signal, training the machine learning with the features set and their associated labels (apnea and normal) for each minute, and finally testing the machine learning performance using cross validation leave-one-out method.

Heart rate variability parameter has attracted the interest of more researchers who employed different methods to extract relevant information. In [6] authors performed sample entropy (SaEn) measure on a perminute basis on HRV of ECG signal. Sample entropy feature was classified using Fisher's Discriminant Function (FDF) on its own which resulted in $70.3 \%$ of accuracy. Power Spectrum Density (PSD) of RR intervals was also computed and a vector of the first 20 features was applied to the classifier. The obtained accuracy with PSD features was not much different from sample entropy's one $(70.3 \%)$. Interestingly, the combination of SaEn and 20 PSD features achieved a higher accuracy of 72.9\%. Here, authors demonstrated that OSA classification performance can be possibly increased when joining two or more different features. However, the accuracy is not as good as what was achieved in CinC challenge [4]. Various features were extracted in both time and frequency domain from RR intervals and ECGDerived Respiration (EDR). Bivariate time-varying autoregressive model (TVAM) was developed in [7] and PSD was calculated as feature for both RR intervals and EDR signal with combination of K-Nearest Neighbour $(\mathrm{KNN})$ and Neural Network (NN) classifiers. This 
resulted in good accuracy performance of $88 \%$ accuracy. Permutation Entropy (PE) was recently applied to RR intervals time series in [8] along with 20 cepstrum coefficients from HRV. A matrix of 20 PSD values was also extracted from EDR signal. In total, 41 features were jointly classified using LR and Quadratic Discriminant Analysis (QDA) techniques. The performance of different features combinations were also investigated. The techniques proposed in this paper provided good OSA detection performance with $71.9 \%$ of sensitivity and $92.1 \%$ of specificity for LR and $75.1 \%$ of sensitivity and $90.5 \%$ of specificity for QDA.

\section{METHODS}

\section{A. The Database}

A total of 35 sleep apnea ECG recordings for Computers in Cardiology Challenge 2000 were obtained from Physionet database [9]. These recordings originate from 32 different individuals of different age and sex who volunteered for previous studies related to OSA. Each recording contains one million samples captured at a rate of 100 samples/second with 16 bit resolution per sample. 35 apnea annotations (labels) were provided for each recording, where each minute was labelled as normal " $N$ " or apnea "A" by human experts through oxygen saturation and respiration signals analysis based on airflow and desaturation amplitudes properties. The recordings are classified into three groups: 20 recordings from "Group A" each containing 100 minutes of apnea or more, 20 recordings from "Group C" containing less than 5 apneic minutes per recording and the remaining 10 recordings belong to "Group B" which is a boundary group where each recording contains between 5 and 99 apneic minutes.

\section{B. Pre-Processing}

An initial analysis of the data was carried out before any processing. This involved checking the data in timedomain plot. This analysis revealed that four recordings in group $\mathrm{C}$ did not provide any information on heart activity. Therefore, only 26 recordings were used in the study. Each ECG recording was first segmented into 1 minute epochs, from which the features will be calculated, since OSA annotations were provided on per-minute basis. Data was cleaned through a number of processes including DC drift cancellation, low pass and high pass filtering by means of Pan-Tompkins algorithm [10]. The latter was eventually used to identify the locations of QRS complexes of ECG signal.

\section{Features Extraction}

Time Sequence Analysis (TSA) was used in electrical field such as partial discharge to diagnose the degradation of the equipment [11]. This method involves the calculation of time difference between the $2^{\text {nd }}$ pulse and first pulse $\left(\Delta \mathrm{T}_{21}\right)$ and between the $3^{\text {rd }}$ pulse and $2^{\text {nd }}$ pulse $\left(\Delta \mathrm{T}_{32}\right)$ of a time series. The delta parameters are represented in 2-dimensional plot. This method is applied to temporal locations of $\mathrm{R}$ peaks where two consecutive RR interval time series (see Fig. 1) of each minute of the
ECG recording are plotted in 2-dimensional as depicted in Fig. 2 and Fig. 3. In order to quantify the relationship between the 2-D parameters, Pearson correlation coefficient was calculated providing one feature per minute. Pearson correlation coefficient is a measure of linear relationship or the degree of co-variance between two variables and can be calculated using the following equation:

$$
\rho=\frac{\operatorname{cov}(x, y)}{\sigma_{x} \sigma_{y}}
$$

where $\operatorname{cov}(x, y)=\sum_{i=1}^{N} \frac{\left(x_{i}-\bar{x}\right)}{\left(y_{i}-\bar{y}\right)}$

$\bar{x}$ and $\bar{y}$ are the mean values of $\mathrm{x}$ and $\mathrm{y}$ vectors respectively.

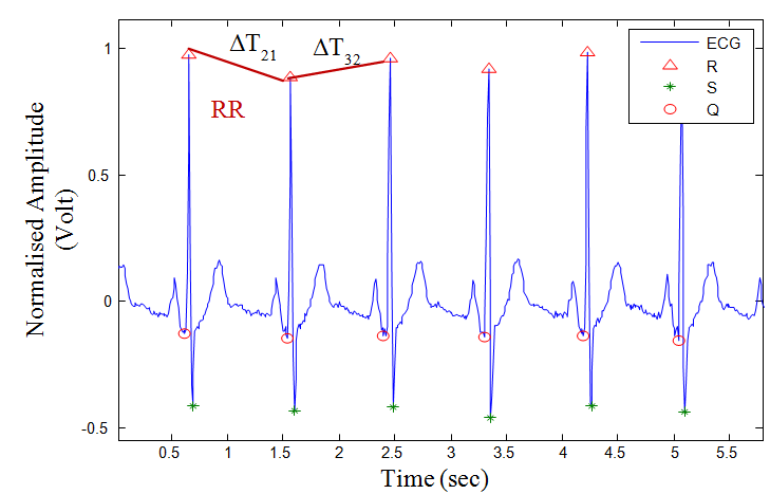

Figure 1. Time sequence analysis and RR intervals of ECG signal.

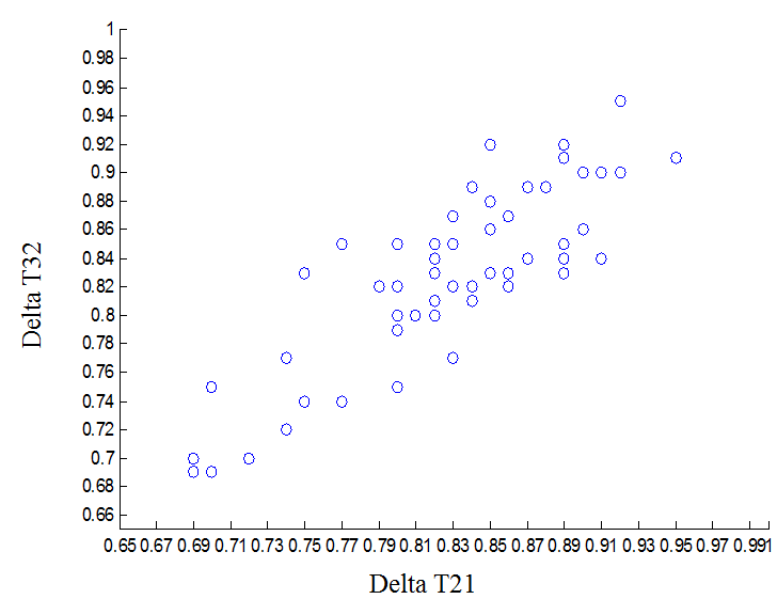

Figure 2. Time sequence analysis plot for normal minute.

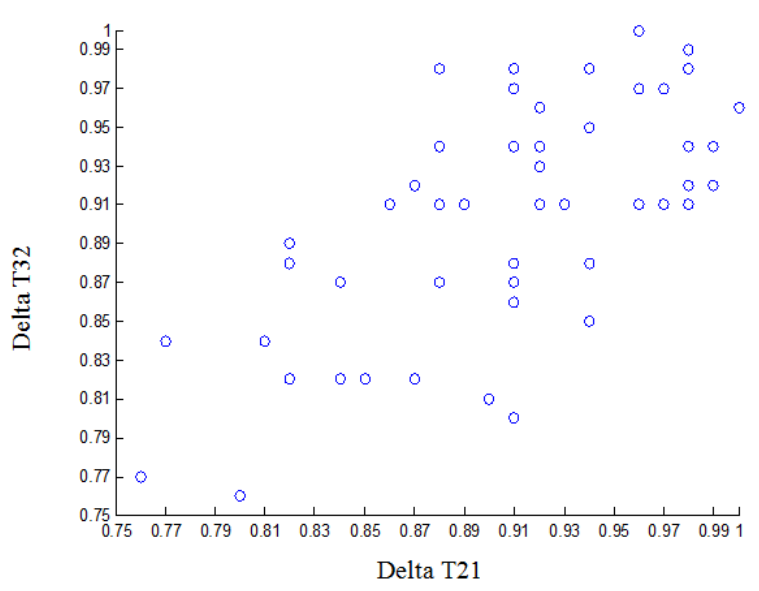

Figure 3. Time sequence analysis plot for apneic minute. 
A correlation coefficient value close or equal to one indicates that the variables are likely to be aligned on the same line. Whereas, a value close or equal to zero means that there is no linear relationship between the variables. A positive value is observed when both variables vectors increase simultaneously. In contrast, a negative value shows that variables of one vector increase while variables of the other vector decrease.

\section{Classification}

Extreme Learning Machine (ELM) is a non-linear single hidden layer feed-forward neural network for large data processing. It supports both regression and multiclass classification. ELM has the advantage of high speed learning, no iterative tuning, low complex implementation and few users' supervision requirement. Various applications such as cloud computing [12], and vehicle routing and gesture identification systems, as cited in [13], exploit ELM for these advantages.

ELM theory is explained in [14] as follow. Given $N$ training input samples $\left(x_{i}, t_{i}\right)$ where $x_{i} \in R^{n}$ is the data vector and $t_{i} \in R^{m}$ is the labels vector. ELM process involves the following three main steps:

1) Assign random weight $w_{i}$ to the input samples where $i=1, \ldots, \tilde{N}$.

2) Compute the hidden-layer output matrix $H$ $=\left[h\left(x_{1}\right), \ldots, h\left(x_{N}\right)\right]^{T}$ using the activation function " $g(x)$ " as expressed in (2).

$$
h\left(x_{i}\right)=g\left(x_{i} \cdot w_{i}\right)
$$

3) Calculate the weight matrix $\beta$ using the expression in (3).

$$
\beta=H^{\dagger} T=\left[\beta_{1}, \ldots, \beta_{L}\right]
$$

where $H+$ is the Moore-Penrose pseudoinverse and $T=$ $\left[t_{1}, \ldots, t_{N}\right]$.

This algorithm operates with a number of activation functions provided by ELM such as sigmoidal, radial basis, sine etc. Given the testing input samples $\left(x_{i}\right)$ with unknown labels, ELM would provide the estimated output based on the calculated $\beta$ in the training phase and on the computed $H$ matrix.

\section{RESULTS}

One feature was extracted per minute per ECG recording from TSA. The feature is computed by Pearson correlation coefficient of $\mathrm{Nx} 2$ vector of $\Delta \mathrm{T}_{21}$ and $\Delta \mathrm{T}_{32}$. Cross validation leave-one-out method was used for training and testing the machine learning algorithm, where 25 subjects were used for training and one subject was left for testing. This process was performed 26 times, leaving different subject in each iteration. The average of all testing accuracies resulting from each iteration was calculated to determine the total OSA diagnosis accuracy. The Nx1 feature vector was implemented to ELM classifier along with labels of each minute for training stage where $\mathrm{N}$ is the total number of minutes in 25 subjects. Similarly, the Mx1 feature vector for one subject was implemented to ELM without the labels to validate the algorithm's performance. This resulted in good and acceptable performance with testing accuracy of $80.3 \%$. In [15] authors performed OSA diagnosis using ELM classification and achieved $87.7 \%$ accuracy. However, they computed a matrix of 68 features which requires increased computation, with respect to our study which only utilises one feature. In [16] authors attempted to detect OSA using two features Power Spectral Density (PSD) and Sample Entropy computed over RR intervals of ECG signal. Fisher's Distribution Function (FDF) method was used for classification. The testing accuracy was lower than the present study, with a percentage of $72.9 \%$. The work detailed within this paper provides acceptable performance with low computational requirements which are suitable for application on low cost hardware when combined with the ELM.

\section{CONCLUSION}

This paper addressed the problem of OSA diagnosis that has serious implications on individual's health and lifestyle. The aim and objectives were achieved successfully. It is found that correlation coefficient of TSA representation, extracted from each minute of ECG recordings is a potential feature for automatic OSA detection with low computation, good enough accuracy which is in line with previous papers. ELM technique can eventually be used as classification algorithm for OSA prediction. As future work, the proposed feature extraction technique could be investigated further to employ different statistical analysis approaches such as applying 3-D representation of three consecutive RR time series, or finding Gaussian mean and standard deviation of 2-D or 3-D time sequences. TSA could also be applied in different fields for monitoring and diagnosis or in applications that require low computation. It could also be applied in different fields for monitoring and diagnosis or in applications that require low computation.

\section{REFERENCES}

[1] H. Yaggi, J. K. Concato, W. N. Kernan, J. Lichtman, L. Brass, and V. Mohsenin, "Obstructive sleep apnea as a risk factor for stroke and death," The New England Journal of Medicine, vol. 353, no. 19, pp. 2034-2041, 2005.

[2] U. R. Abeyratne, S. Silva, C. Hukins, and B. Duce, "Obstructive sleep apnea screening by integrating snore feature classes," Physiological Measurement, vol. 34, no. 2, pp. 99-121, 2013.

[3] O. Parra, A. Arboix, and S. Bechich, "Time course of sleep-related breathing disorders in first-ever stroke or transient ischemic attack," AM. J. Respir. Crit. Care Med., vol. 161, pp. 375-380, 2000.

[4] T. Penzel, J. McNames, P. D. Chazal, B. Raymond, A. Murray, and G. Moody, "Systematic comparison of different algorithms for apnoea detection based on electrocardiogram recordings," Medical \& Biology Computing \& Engineering, vol. 40, pp. 402-407, 2002.

[5] M. O. Mendez, A. M. Bianchi, M. Matteucci, S. Cerutti, and T. Penzel, "Sleep apnea screening by autoregressive models from a single ECG lead," IEEE Transactions on Biomedical Engineering, vol. 5, no. 12, pp. 2838-2850, 2009.

[6] C. Marchesi, M. Paoletti, and S. D. Gaetano, "Global waveform delineation for RR series estimation: Detecting the sleep apnea pattern," Computer Cardiology, vol. 27, p. 71, 2000.

[7] D. Avarez, R. Hornero, J. Marcos, F. Campo, and M. Lopez, "Spectral analysis of electroencephalogram and oximetric signals in obstructive sleep apnea diagnosis," in Proc. 31st IEEE International Conference on Engineering in Medicine and Biology Society, 2009, pp. 400-403. 
[8] F. Ng, I. Garcia, P. Gomis, A. L. Cruz, G. Passariello, and F. Mora, "Bayesian hierarchical model with wavelet transform coefficients of the ECG in obstructive sleep apnea screening," Computer Cardiology, vol. 27, pp. 275-278, 2000.

[9] A. L. Goldberger, et al., "PhysioBank, PhysioToolkit, and PhysioNet: Components of a new research resource for complex physiologic signals, Circulation, vol. 101, no. 23, pp. e215-e220, 2000.

[10] J. Pan and W. J. Tompkins, "Real time QRS detector algorithm," IEEE Transaction in Biomedical Engineering, vol. 32, no. 3, pp. 230-236, 1985.

[11] B. Stewart and L. Yang, "A simple metric for assessing the severity of partial discharge activity based on Time-SequenceAnalysis discharge level patterns," Transactions on Electrical and Electronic Materials, vol. 7, no. 6, pp. 313-318, 2006.

[12] J. Lin, J. Yin, Z. Cai, Q. Liu, and K. Li, "A secure and practical mechanism for outsourcing ELMs in cloud computing," IEEE Intelligent Systems, vol. 28, no. 6, pp. 35-38, 2013

[13] E. Cambria and G. Huang, "Exreme learning machines," IEEE Intelligent Systems, vol. 28, no. 6, pp. 30-34, 2013

[14] G. Huang, "Extreme learning machine," IEEE Computer Society, vol. 13, pp. 30-59, 2013.

[15] N. Sadr and P. D. Chazal, "Automated detection of obstructive sleep apnoea by single-lead ECG through ELM classification," in Proc. Computing in Cardiology Conference, Cambridge, MA, 2014.

[16] H. Al-Angari and A. Sahakian, "Use of sample entropy approach to study heart rate variability in obstructive sleep apnea syndrome," IEEE Transactions on Biomedical Engineering, vol. 54, no. 10, pp 1900-1904, 2007.

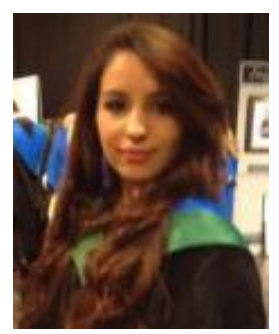

Imene Mitiche obtained a BSc. in Computer and Electronic Systems Engineering (Software development) with first class honours and a MSc. in Telecommunications Engineering with distinction from Glasgow Caledonian University. She is currently pursuing her PhD at Glasgow Caledonian University. Her area of research is signal processing and machine learning. Her other interests include modern communication systems and programming.

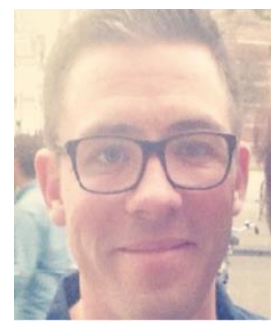

Gordon Morison received a BEng in Electrical and Electronic Engineering, an $\mathrm{PhD}$ in Signal and Image Processing from the University of Strathclyde. $\mathrm{He}$ has held industrial positions in embedded software and post-doctoral positions in Communications at the University of Strathclyde and in Neuroscience at the University of Glasgow. Currently he is a Lecturer in Computer, Communications and Interactive systems at Glasgow Caledonian University. His research interests are in Signal/Image Processing and Machine Learning.

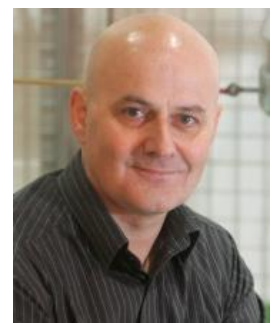

Brian G. Stewart obtained B.Sc. and Ph.D degrees from the University of Glasgow, Glasgow, U.K., and a B.D. degree from the University of Aberdeen, Aberdeen, U.K. He is a Professor in the School of Engineering and Built Environment at Glasgow Caledonian University, Glasgow, U.K. He has been involved in the research, development, and application of partial discharge instrumentation and insulation diagnostics techniques for High Voltage systems for the past 14 years. He also has research interests in the field of communication systems. Prof. Stewart is currently an Ad Com Member of the IEEE Dielectrics and Electrical Insulation Society, a Chartered Engineer, and a Member of the IET. 